\title{
Biosensors Technologies for Point-of-Care Testing - A Review
}

\author{
Sylwia Baluta ${ }^{1 *}$, Joanna Cabaj', Karol Malecha² \\ ${ }^{1}$ Department of Organic and Medicinal Chemistry, Faculty of Chemistry, Wrocław University of Science and Technology, \\ 50-370 Wrocław, 27 Wybrzeże Wyspianskiego st., Poland \\ ${ }^{2}$ Department of Microsystems, Faculty of Microsystem Electronics and Photonics, Wrocław University of Science and Technology, \\ 50-370 Wrocław, 27 Wybrzeże Wyspianskiego st., Poland \\ * Corresponding author, e-mail: sylwia.baluta@pwr.edu.pl
}

Received: 29 November 2019, Accepted: 08 April 2020, Published online: 17 September 2020

\begin{abstract}
Chronic illnesses require continuous monitoring and medical intervention for efficient treatment to be achieved. Therefore, designing a responsive system that will reciprocate to the physicochemical changes may offer superior therapeutic activity In this respect, biosensors development, which offers constant, fast, selective and sensitive in situ monitoring is extremely important. The ability of biosensors miniaturization opens new technological pathways for the development of innovative approaches, which will be able to detect a wide range of compounds in the "multi-mode" system. Miniaturization and integration of important components result in many advantages: reducing the time of analysis and laboratory processes, automatization of measurements, compactness, and portability. Biosensing instruments also represent a very promising scientific way for construction new generation of wearable, portable and implantable bioelectronic devices for Point-Of-Care (POC) testing application. Biosensors also offer a powerful opportunity in early diagnosis and treatment of illness, which is an essential value in the case of POC testing. POC is mostly focused on the patient with chronic illness, where the continuous monitoring of analytes, is required to allow changing of the dosage and treatment period. In this review, we present the application of biosensing platforms in one chip, which can be used in wireless, wearable and swallowable sensors for POC diagnostic.
\end{abstract}

Keywords

biosensors, implantable sensors, Point-Of-Care (POC) testing, swallowable sensors, wearable sensors, wireless sensors

\section{Introduction}

Chronic illnesses require continuous monitoring and medical intervention for efficient treatment to be achieved. Therefore, designing a responsive system that will reciprocate to the physicochemical changes may offer superior therapeutic activity [1]. In this respect, biosensors development, which offers constant, fast, selective and sensitive in situ monitoring is extremely important. Biosensors belong to the group of chemical sensors, which are analytical devices that use biological recognition elements (such as enzymes or antibodies) for the detection of disease biomarkers, followed by the quantification of biomarkers using transducers (Fig. 1) [2-4].

Since 1962, when Leland Clark introduced the first biosensor prototype, biosensors became omnipresent in a wide range of important areas such as biomedical diagnosis, point-of-care monitoring of treatment and disease progression, environmental monitoring, food control, drug discovery, forensics and biomedical research [5]. A biosensor is composed of two intimately associated elements: a receptor layer and a transducer. The receptor layer is built of biological material (e.g. enzymes, whole cells, antibodies) and recognizes the analyte, whereas the transducer converts the (bio)chemical signal resulting from the interaction of the analyte with the bioreceptor into a digital electronic signal [6]. The output signal is directly or inversely proportional to the concentration of the examined substance. Their connection in the bioreceptor part with high-affinity biomolecules allows the sensitive and selective detection of a range of analytes. One of the most important applications of biosensors is the point of care testing, which is to be the primary form of diagnostic for many patients in the developing world.

Point-Of-Care (POC) testing is generally defined as tests that are performed away from the medical laboratory. There is still not any universal definition of such testing however, any tests that can be done quickly, simply and without the need for sophisticated laboratory equipment is defined as POC. According to Peeling et al. [7] 


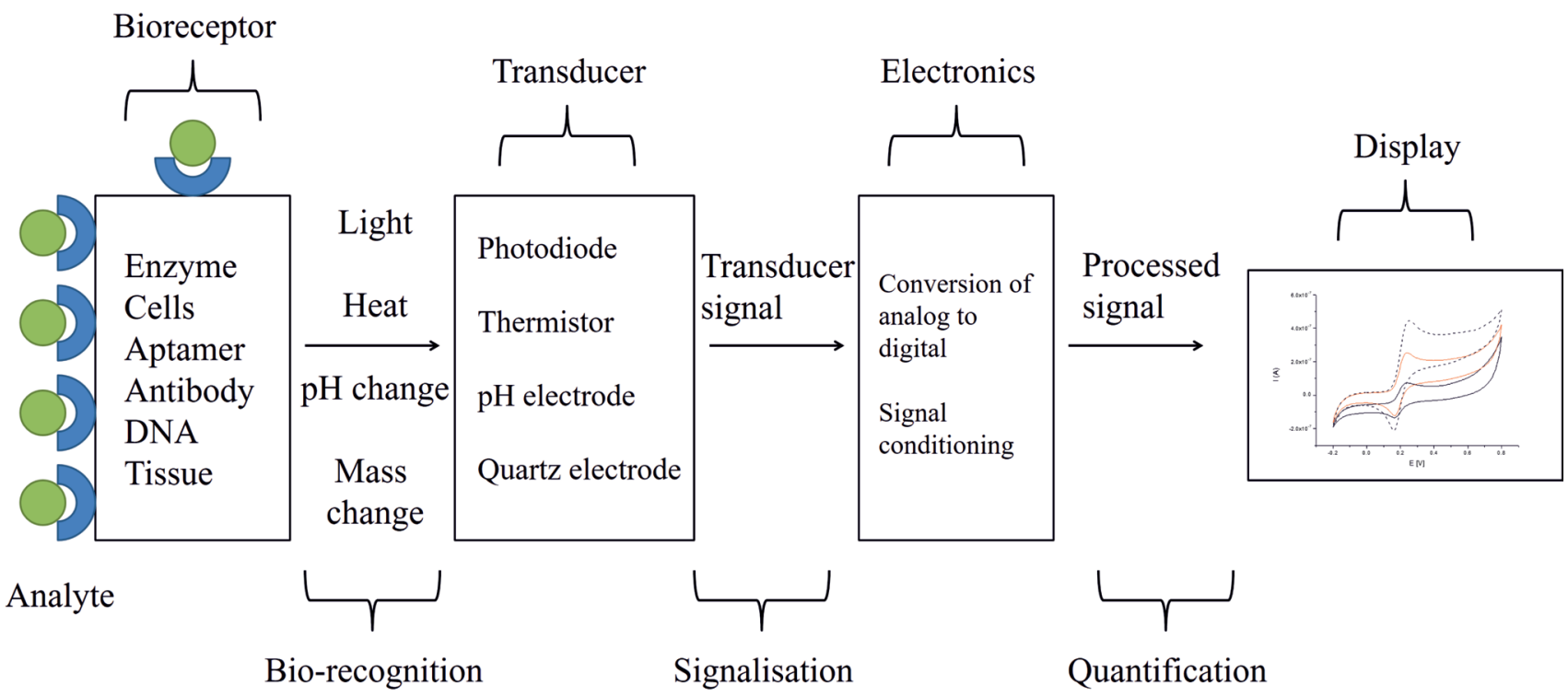

Fig. 1 Schematic description of biosensor work (according to [4])

the fundamental requirements for POC are accuracy, sensitivity, specificity, user-friendliness, rapid and robust, equipment-free and delivered (ASSURED) at settings away from traditional laboratory. In comparison with conventional analytical testing POC possess many advantages, they are more efficient, the turnaround time for results is decreased, which is directly connected with faster patient diagnosis, allow for rapid response and continuous monitoring of critical parameters, they are portable and more convenient, indirect costs by avoiding travel can be reduced [8].

Biosensors, thank to fast measurement, offer a powerful opportunity in early diagnosis and treatment of illness, which is an essential value in case of point-ofcare testing. Additionally, they can be fully automated and miniaturized. POC is mostly focused on the patient with chronic illness, such as Alzheimer's or Parkinson's diseases, diabetes or epilepsy where the continuous monitoring of important analytes is required to allow changing of the dosage and treatment period. Wireless POC systems (mostly biosensors) provide the continuous measurements and monitoring of important biological parameters connected with such disorders [9, 10]. These bio-analytical systems can be divided into two groups: implantable biosensors and wearable biosensors.

Implantable biosensors become more interesting, due to their cost efficiency and diversity of application. Connection of biosensors with wireless capability provides real-time monitoring of health conditions, and also control of real-time drug delivery systems. In the future, such systems will be miniature sensors equipped with a single platform that could measure a lot of different parameters, such as $\mathrm{pH}$, pressure, temperature. The integration of biosensors and Integrated Circuit (IC) technology with a wireless link forms the basis for future multisensor microsystems and leads to real-time and continuous monitoring of chronic illness [11].

Wearable biosensors are mostly placed in patients' outfit or skin (as a tattoo) and are also used for permanent control of some vital health parameters. For instance, a wristworn system for monitoring of electrocardiogram (ECG), blood pressure, blood oxygen saturation, acceleration, and temperature, due to connection of all sensors, signal processing, and cellular communication [12].

In this work, wireless biosensors application in pointof-care medical testing is presented.

\section{Diagnostic application of wireless sensors}

Biosensors and sensors have become important diagnostic devices, engage in almost every field of industry, due to their possibilities of miniaturization and automatization. Last, huge progress in nanotechnology has led to develop systems that are implantable, swallowable, wearable, or portable and provide more comfort for patients, with the absence of wires reducing costs and providing more flexibility [13]. Due to this fact, the application of such apparatus possesses many diverse applications, mostly in medical diagnostics. Wearable platforms integrated with chemical or biochemical sensors are a very promising way in the context of the creation of new non-invasive healthcare devices. Wearable electronic devices such as 
smartwatches, smart glass, and wearable cameras present a wide range of applications in healthcare monitoring. They permit continuous analytes monitoring, which provides detailed information unattainable by intermittent blood sampling, including real-time, "24/7" display of analyte level and rate of changes [14]. Many constructed systems are using the "smart materials", such as graphene, nanoparticles, which are focused on the monitoring of biological fluids, for instance, interstitial fluids, breath, sweat, saliva and tears [15].

\subsection{Wireless wearable sensors}

For appropriate performing real-time, continuous monitoring, using the wireless sensor, of important parameters in the patient's body must be taken into account three main features of such devices: battery-less operating mode for long-term use and preservation of the integrity of the sensed/stimulated tissues, respectively [16]. Unfortunately, due to the fact, that it is extraordinarily hard to counteract wireless system/skin because of the interface features cumbersome time-varying dynamics [17]. The article presented in Science shows the soft elastomeric substrate in microfluidic assemblies. They present structured adhesive surfaces and controlled mechanical buckling to achieve ultralow modulus, highly stretchable systems that incorporate assemblies of high-modulus, rigid, state-of-the-art functional elements [18]. Such a wearable, flexible device can monitor heart rate and other crucial health parameters.

The Connection of nanosensors with biomaterials may present a high impact on the monitoring of health parameters and adaptive threat detection. Due to the nanoscale nature of graphene, this material is suitable for very sensitive detection of analytes [19]. Mannoor et al. [20] presented the method for printing graphene onto water-soluble silk. Such printing allows for direct biotransfer of graphene nanosensors onto biomaterials, such as tooth enamel. In the consequence, the scientists created a biointerfaced sensing system for selective bacteria detection in saliva, due to self-assembly of antimicrobial peptides onto graphene. The sensing platform is based on graphene connected with a wireless readout coil that is generated on silk fibroin. Then, the thin nanosensors are directly biotransferred from the silk platform onto biomaterials, such as tooth enamel, via dissolution of the supporting silk film. The large surface area of the graphene and electrodes ensures high adhesive conformability to the rugged surfaces of biomaterials. Biological selectivity was obtained due to AMP-graphene peptides self-assembling onto the graphene monolayer. Such a tattoo-like bio-platform could detect even one cell bacteria, so the measuring system is very sensitive. Now, we present some analytes, which can be detected with the described above techniques.

\subsection{Glucose monitoring}

The first biosensor, for detecting glucose directly in a sample, was described in 1962 [21]. Since then, intensive researches are underway to improve existing sensors and to develop new ones - more accurate and sensitive. However, the most popular are glucometers - indispensable for the determination of blood glucose levels in patients with diabetes. Many pharmaceutical companies provide glucose meters, such as: in Poland - Diagnostic System Gold (Diagnosis), in Germany - Contour TS (Bayer) or in the USA - Precision Xtra (Abbott Diabetes Care Ltd.). Moreover, there are also a few devices, allowing for glucose monitoring without the need for finger pricking. An epidermal electrochemical glucose monitoring is examined using two bio-fluids: skin interstitial fluid (ISF) and sweat. The concentration of glucose in sweat is a little bit smaller than the glucose level in the blood, because of the diffusion process of this analyte from the blood vessels through sweat glands [22, 23]. Certain solutions are based on direct implantation through a needle (e.g. Guardian RT by Medtronic) [24] while others employ a microdialysis harvesting approach (e.g. GlucoDay by Menarini [25]) correlated with external monitoring of the collected sample.

Weinstein et al. [26] presented the FreeStyle Navigator Continuous Glucose Monitoring System, which is construct based on four elements: a miniature electrochemical sensor placed in the subcutaneous adipose tissue, a disposable sensor delivery unit, a radiofrequency transmitter that connects to the sensor, and a hand-held receiver to receive the sensor signal and display continuous glucose values. The purpose of the sensor is measuring the level of the glucose in the interstitial fluid, where the glucose concentration corresponds to the concentration of this analyte in a patient's blood. The monitoring of glucose is based on enzymatic reaction using glucose oxidase and uses an osmium mediator covalently bound to the polymer matrix [26]. Such a device can be used for 5 days and it is a single-use element.

Another example of a wearable sensor for glucose monitoring is a system described by Kudo et al. [27]. The biosensor was designed using microelectromechanical system (MEMS) technique (the fabrication of the electrodes) combine with the functional polymer membranes based on 
polydimethylsiloxane (PDMS) and 2-methacryloyloxyethyl phosphorylcholine (MPC) copolymerized with dodecyl methacrylate (DMA). Onto a hydrogen peroxide, Pt working electrode has been also immobilized a glucose oxidase (Fig. 2). Flexible biosensor allows for the current measurements based on redox reaction catalyzed by the enzyme. The created biosensor was optically transparent except the electrodes, and it can trace the rounded shape of the finger. Additionally, the designed sensor was stretchable [27].

Another new wearable analytical device, demonstrated by Lee et al. [28], is biosensor allowing for glucose monitoring in sweat. The glucose sensor is based on a Prussian blue-covering on a gold electrode and glucose oxidase, measurements are provided due to the galvanostatic method, and it can control sweat through impedance changes of poly(3,4-ethylenedioxythiophene) (PEDOT) interdigitated electrodes. Additionally described a system for sweat glucose monitoring is integrated with feedback transdermal drug delivery by hyaluronic acid hydrogel microneedles. Such miniaturized wearable biosensor can sensitive detect glucose from even $1 \mu \mathrm{L}$ of sweat in a wide range of concentrations: $10 \mu \mathrm{M}-1 \mathrm{mM}$.

\subsection{Ethanol monitoring}

The evolution of gas sensors for ethanol monitoring was driven by the need for faster and more versatile analytical methods for application in important areas including clinical, diagnostics, food analysis, environmental monitoring and industry analysis. One of the key problems in commercially available devices for ethanol monitoring showing issues from long-term fouling of the sensor element and drift in the resulting sensor readings over time [29]. Here, we present recently presented gas sensors for ethanol surveys, which can provide measurements at room temperature.

Zheng et al. [30] introduced in Scientific Reports a flexible and transparent gas sensor for ethanol monitoring designed for wearable applications. An ITO (Indium Tin Oxide) was coated using PET (polyethylene terephthalate), and on such substrate was then used a method of drop-casting of $\mathrm{ZnO}$ nanoparticles onto PET-ITO surface. The photoelectric and current versus voltage (I-V) curves were studied using a Keithley 4200-SCS semiconductor characterization system (the transducer). Ethanol was measured based on the flow-through procedure.

A different system based on modified with multiwall carbon nanotube (MWCNTs) and polyvinyl alcohol (PVA) cotton fabrics appeared in Sensors and Actuators B, described by Maity et al. [31], presented VA/MWCNTs coated cotton fabric sensor allows for ethanol monitoring at room temperature and is characterized by high flexibility. Mechanism of sensing ethanol is based on a change in intertube contact resistance of PVA/MWCNTs due to ethanol vapor interaction and diffusion following by swelling of PVA layers. Presented results are very promising in case of developing new, flexible, room temperature operable wearable gas sensing platforms. The gas sensor presents good linearity in the concentration of ethanol vapors in range $100-500 \mathrm{ppm}$ and the detection limit was found $9.17 \mathrm{ppm}$.

Kim et al. [32] designed a wearable tattoo-based biosensing system for ethanol monitoring in sweat. In this novel approach, scientists construct a wearable platform leans on the connection of an iontophoretic-biosensing temporary tattoo system with flexible wireless electronics. The principle of detection in the proposed approach uses constant-current iontophoresis for inducing sweat by supplying the drug pilocarpine through the skin, followed by amperometric biosensing of the ethanol present in sweat. An amperometric measurement uses the catalytic redox reaction of ethanol by an alcohol-oxidase (AOx) enzymatic electrode along with a printed Prussian Blue (PB) electrode transducer. The system, presented by Kim et al. [32], has been utilized on human objects and has found to be very sensitive during ethanol measurements.

\subsection{Metals monitoring}

Despite electrolytes, metabolites, proteins in body fluids are also present heavy metal ions, which are very often reflecting patient's health conditions [33]. Accumulation
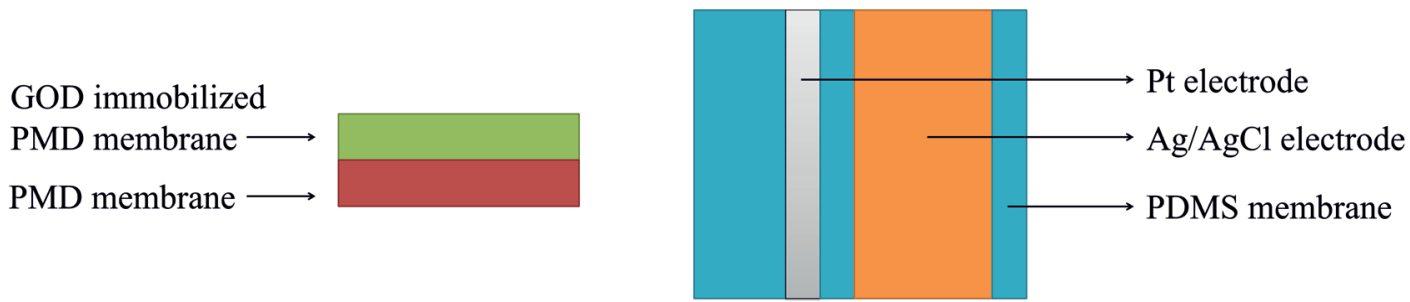

Fig. 2 General scheme of construction of electrodes based on MEMS technique with polymer membranes allowing for glucose investigations 
of heavy metal ions may lead to numerous serious disorders, just like a deficiency. Unfortunately, the metal ions monitoring meets with the problem of concentration levelin the human body proper level of metal ions is in the order of $\mu \mathrm{g} / \mathrm{L}$. However, it is extremely important to detect these analytes, because of the huge effect on our health. For instance, Wilson's disease is connected with copper concentration [34] and, what is more, any aberration in copper level influence on the proper working of vital organs, such as kidney, heart, liver, brain, additionally lower concentration lead to anemia and osteoporosis [33]. Excess of cadmium and mercury in the human body have a damaging effect on nervous, immunological and cardiovascular systems [35]. Low zinc level may result in occurring pneumonia or diarrhea, on the other hand to many zinc in the patient's body lead to liver damage [36]. Maintaining balance in the concentration of metal ions is a crucial challenge in modern medicine and POC testing, due to daily exposure to metals effect due to environmental pollution.

Gao et al. [37] presented in their work synchronous method for multiplexed detection of heavy metal ions concentration in body fluids (sweat and urine) (Fig. 3).

A wearable microsensor designed for monitoring $\mathrm{Zn}, \mathrm{Cd}$, $\mathrm{Pb}, \mathrm{Cu}$, and $\mathrm{Hg}$ is based on a survey of an electrochemical square wave anodic stripping voltammetry (SWASV) on Au and Bi microelectrodes. Obtained oxidation peaks were calibrated and compensated due to using a skin temperature sensor. Microsensor exhibited excellent selectivity, repeatability, and flexibility parameters, which is very important in the case of designing POC testing analytical systems.

Suitable for wearing a sensor for calcium ions detection is described by Nyein et al. [38] in ACS Nano. Scientists focused on development electrochemical noninvasive, wearable and flexible system for real-time monitoring of $\mathrm{Ca}$ ions and changes in $\mathrm{pH}$ in patients' tears, sweat, and urine. $\mathrm{Ca}^{2+}$ and $\mathrm{pH}$ measurements are conducted based on an electrochemical platform combining skin temperature, $\mathrm{pH}$ and $\mathrm{Ca}^{2+}$ sensors made from flexible PET.
The level of calcium ions and $\mathrm{pH}$ changes uses ion-selective electrodes connected with a polyvinyl butyral (PVB)-coated $\mathrm{Ag} / \mathrm{AgCl}$ reference electrode. Electrical potential differences between these two electrodes lead to obtaining proportional to the logarithmic concentration of respective target ions and are measured with the aid of the interfacing signal conditioning circuitry. Additionally, the system is equipped in sensing electrodes, responsible for receiving the signals:

- $\mathrm{Ca}^{2+}$ electrode is designed based on an organic membrane containing electrically neutral carrier calcium ionophore II and an ion-electron transducer

- $\mathrm{pH}$ electrode can detect hydrogen ions due to the deprotonation process at the surface of polyaniline (PANI).

Signals corresponding to a given concentration are generally read and processed by a microcontroller. Then, all data are transmitted through Bluetooth to a mobile phone and are displayed on it (Fig. 4, [38]).

\subsection{Wireless implantable sensors}

The next type of wireless sensors are an implantable sensors. The biggest challenges connected with designing wireless sensing systems that can be implanted in the living organisms are size, power consumption, functionality, and reliability of these devices. The integration of miniaturized electronics is a key step to manage with such stringent requirements [39]. What is more, an implantable device must be biocompatible with the tissue, due to this fact, bioactive and biomimetic materials are widely investigated to induce proper tissue responses. The main goal of implantable devices is to improve the life quality of the patients, who are suffering in chronic diseases and must permanently use of bioanalytical and invasive tests. Since now, commercially available are devices for the management of brain and heart diseases [40, 41]. Lewitus et al. [42] proposed a new strategy for construction bioactive composites based on polysaccharide agarose and carbon-nanotube (A-CNE), which

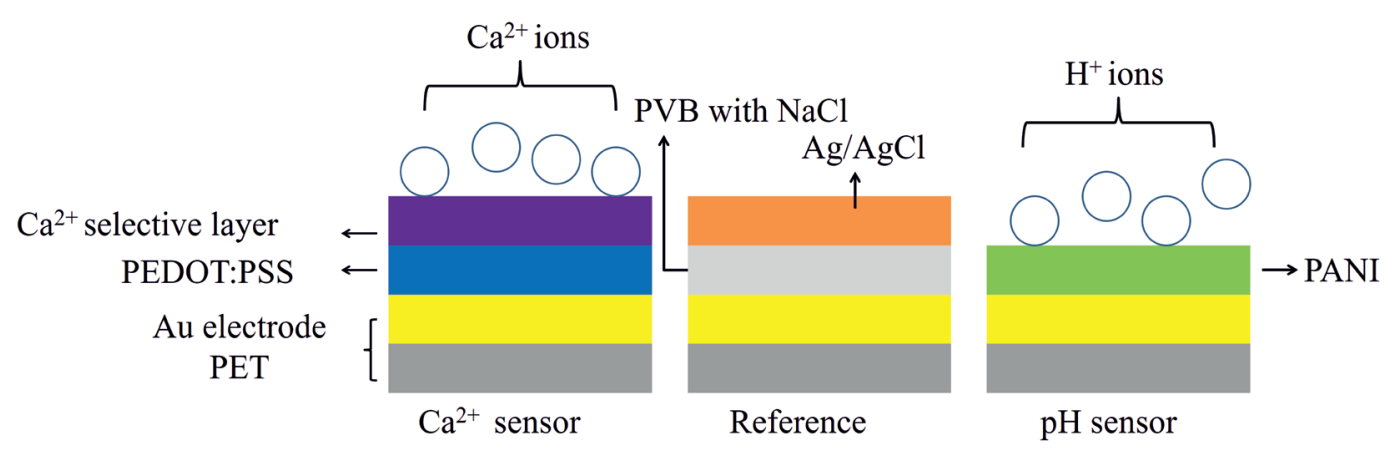

Fig. 3 General scheme of the method for multiplexed metal ions determination (based on [38]) 


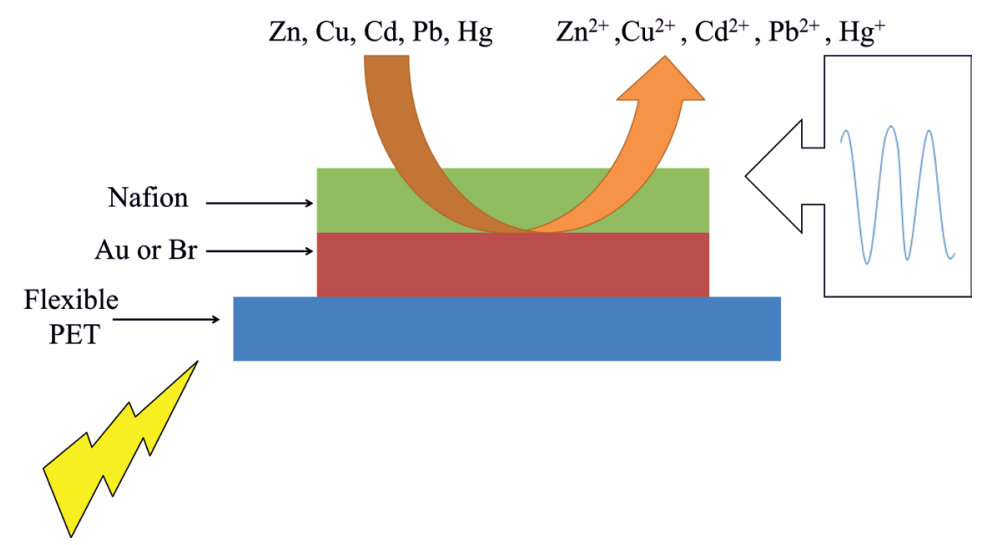

Fig. 4 General scheme of the working method of an electrochemical system for calcium ions monitoring in the presence of $\mathrm{H}^{+}($based on [38])

can manipulate interface brain-implant. Such a system can be applied as a microelectronic instrument that is biocompatible with the brain tissue. A-CNEs were functionalized by conjugating with neuro-relevant proteins - laminin and alpha-melanocyte stimulating hormone and successfully implanted in the brain. Examination of neuronal and immunological responses (qualitative and quantitative) showed characteristic changes depending on the type of biomolecule used. The potential to manipulate tissue response through the use of an anti-inflammatory protein, alpha-melanocyte stimulating hormone, was shown in the reduction of astroglia presence near the implant site during the glial scar formation. Obtained results may lead to the development of novel surface modifications of carbon nanotubes-based platforms to improve the efficiency of implantable devices.

A similar type of such approach is a swallowable sensor, which also should work inside of the patient body, however not continuously.

\subsection{Swallowable sensors}

The main goal of designing the swallowable sensors is to investigate the entire gastrointestinal (GI) tract for a wide range of diseases, such as tumors, cancer, ulcers, Crohn disease, GI bleeding and celiac disease. Classical techniques, for instance: ultrasonography (USG), X-radiography (X-ray) or computer tomography (CT), despite many advantages do not permit observation of the whole GI tract, because of its complex, curvy architecture [43]. Conventional gastroscopy and colonoscopy, using probe endoscopy is very often a painful and causing discomfort method. Apart from the capsule technology that has been developing since the 1950s, a breakthrough occurred in 2000 [44, 45]. Iddan et al. [45] in Nature presented invention and examination of a new type of video-telemetry capsule endoscope, which was able to record the inspection for around 5 hours, was small enough to be swallowed $\left(11 \times 30 \mathrm{~mm}^{2}\right)$ and had no external wires, fibre-optic bundles or cables. To construct such a wireless video system, the scientists combined three technologies: complementary metal-oxide-semiconductor (CMOS) image sensors, application-specific integrated circuit (ASIC) devices, and white light-emitting diode (LED) illumination. All obtained video images and results were transmitted by UHF-band radio-telemetry. What is most desirable from the patient's point of view, such a technique does not require a hospital environment. Nowadays, there are few clinical capsules present on the market: PillCam SB, PillCam Eso, OMOM, Olympus, MiroCam [46-49].

Currently, the development in wireless endoscopic capsules pays attention to designing new, miniaturized medical robots with wide functions, which could highly improve available capsules. Future capsules could be equipped in the incorporation of mini-surgical tools and even artificial intelligence. To efficiently identify diseases, high-quality images and longer capsule lifetimes are essential [43].

\section{Conclusions}

In this review, we characterized the new trends in biosensoric systems evolution for POC diagnostic. Due to the primary goals of world-wide scientific endeavours, which are based with improvement of the quality of life, the development of new analytical devices is crucial. Achieving this assumption is directly related to the rapid analysis of common disorders, quality control in the food industry and environment monitoring. The dynamic investigation of biosensors for POC technology in recent years and mutual cooperation of several scientific disciplines biology, chemistry, electronics and material engineering, enables the development and upgrading of research tools and measurement for quick and sensitive detection. The most promising technology pathway are wireless 
sensors, which strongly reduce the time and costs of analysis, what is more, this instruments are compact and portable. Mostly, it is important for the field of medical diagnostics, where the availability of a rapid, simple, low-cost, in situ whole-blood assay capable of detecting a variety of selected analytes would benefit POC or public health applications. Wireless sensor technology based on the devices which are implantable, swallowable, wearable, or

\section{References}

[1] Ngoepe, M., Choonara, Y. E., Tygai, C., Tomar, L. K., du Toit, L. C., Kumar, P., Ndesendo, V. M. K., Pillay, V. "Integration of Biosensors and Drug Delivery Technologies for Early Detection and Chronic Management of Illness", Sensors, 13(6), pp. 7680-7713, 2013.

https://doi.org/10.3390/s130607680

[2] Vo-Dinh, T. "Nanosensing at the single cell level", Spectrochimica Acta B: Atomic Spectroscopy, 63(2), pp. 95-103, 2008. https://doi.org/10.1016/j.sab.2007.11.027

[3] Sethi, R. S. "Transducer aspects of biosensors", Biosensors and Bioelectronics, 9(3), pp. 243-264, 1994. https://doi.org/10.1016/0956-5663(94)80127-4

[4] Howes, O., McCutcheon, R., Stone, J. "Glutamate and dopamine in schizophrenia: an update for the $21^{\text {st }}$ century", Journal of Psychopharmacology, 29(2), pp. 97-115, 2015. https://doi.org/10.1177/0269881114563634

[5] Bhalla, N., Jolly, P., Formisano, N., Estrela, P. "Introduction to biosensors", Essays in Biochemistry, 60(1), pp. 1-8, 2016. https://doi.org/10.1042/EBC20150001

[6] Sassolas, A., Blum, L. J., Leca-Bouvier, B. D. "Immobilization strategies to develop enzymatic biosensors", Biotechnology Advances, 30(3), pp. 489-511, 2012.

https://doi.org/10.1016/j.biotechadv.2011.09.003

[7] Peeling, R. W., Holmes, K. K., Mabey, D., Ronald, A. "Rapid tests for sexually transmitted infections (STIs): the way forward", Sexually Transmitted Infections, 82(5), pp. v1-v6, 2006. https://doi.org/10.1136/sti.2006.024265

[8] St John, A., Price, C. P. "Existing and Emerging Technologies for Point-of-Care Testing", The Clinical Biochemist Reviews, 35(3), pp. 155-167, 2014.

[9] Ghafar-Zadeh, E. "Wireless Integrated Biosensors for Point-of-Care Diagnostic Applications", Sensors, 15(2), pp. 3236-3261, 2015. https://doi.org/10.3390/s150203236

[10] Wang, P., Kricka, L. J. "Current and Emerging Trends in Pointof-Care Technology and Strategies for Clinical Validation and Implementation", Clinical Chemistry, 64(10), pp. 1439-1452, 2018. https://doi.org/10.1373/clinchem.2018.287052

[11] Christodouleas, D. C., Kaur, B., Chorti, P. "From Point-of-Care Testing to eHealth Diagnostic Devices (eDiagnostics)", ACS Central Science, 4(12), pp. 1600-1616, 2018. https://doi.org/10.1021/acscentsci.8b00625

[12] Beach, C., Krachunov, S., Pope, J., Fafoutis, X., Piechocki, R. J., Craddock, I., Casson, A. J. "An Ultra Low Power Personalizable Wrist Worn ECG Monitor Integrated With IoT Infrastructure", IEEE Access, 6, pp. 44010-44021, 2018. https://doi.org/10.1109/ACCESS.2018.2864675 portable represents a very promising and prosperous scientific way in this context. We believe, that in the future, POC devices could be able to perform analysis virtually anywhere and under field conditions.

\section{Acknowledgment}

The authors gratefully acknowledge Wroclaw University of Science and Technology for financial support.

[13] Wang, S., Chinnasamy, T., Lifson, M. A., Inci, F., Demirci, U. "Flexible Substrate-Based Devices for Point-of-Care Diagnostics", Trends in Biotechnology, 34(11), pp. 909-921, 2016. https://doi.org/10.1016/j.tibtech.2016.05.009

[14] Kim, J., Campbell, A. S., Wang, J. "Wearable non-invasive epidermal glucose sensors: A review", Talanta, 177, pp. 163-170, 2018. https://doi.org/10.1016/j.talanta.2017.08.077

[15] Vigneshar, S., Senthilkumaran, B. "Current technological trends in biosensors, nanoparticle devices and biolabels: Hi-tech network sensing applications", Medical Devices \& Sensors, 1(2), Article Number: e10011, 2018. https://doi.org/10.1002/mds3.10011

[16] Heikenfeld, J., Jajack, A., Rogers, J., Gutruf, P., Tian, L., Pan, T., Li, R., Khine, M., Kim, J., Wang, J., Kim, J. "Wearable sensors: modalities, challenges, and prospects", Lab on Chip, 18(2), pp. 217-248, 2018. https://doi.org/10.1039/C7LC00914C

[17] Windmiller, R. J., Wang, J. "Wearable Electrochemical Sensors and Biosensors: A Review", Electroanalysis, 25(1), pp. 29-46, 2013. https://doi.org/10.1002/elan.201200349

[18] Xu, S., Zhang, Y., Jia, L., Mathewson, K. E., Jang, K. I., Kim, J., Fu, H., Huang, X., Chava, P., Wang, R., Bhole, S., Wang, L., Na, Y. J., Guan, Y., Flavin, M., Han, Z., Huang, Y., Rogers, J. A. "Soft Microfluidic Assemblies of Sensors, Circuits, and Radios for the Skin", Science, 344(6179), pp. 70-74, 2014. https://doi.org/10.1126/science.1250169

[19] Huang, H., Su, S., Wu, N., Wan, H., Wan, S., Bi, H., Sun, L. "Graphene-Based Sensors for Human Health Monitoring", Frontiers in Chemistry, 7, Article Number: 399, 2019. https://doi.org/10.3389/fchem.2019.00399

[20] Mannoor, M. S., Tao, H., Clayton, D., Sengupta, A., Kaplan, D. L., Naik, R. R., Verma, N., Omenetto, F. G., McAlpine, M. C. "Graphene-based wireless bacteria detection on tooth enamel", Nature Communications, 3(1), Article Number: 763, 2012. https://doi.org/10.1038/ncomms1767

[21] Clark Jr., L. C., Lyons, C. "Electrode Systems for Continuous Monitoring in Cardiovascular Surgery", Annals of the New York Academy of Sciences, 102(1), pp. 29-45, 1962. https://doi.org/10.1111/j.1749-6632.1962.tb13623.x

[22] Zierler, K. "Whole body glucose metabolism", American Journal of Physiology-Endocrinology and Metabolism, 276(3), pp. E409-E426, 1999. https://doi.org/10.1152/ajpendo.1999.276.3.E409 
[23] Cengiz, E., Tamborlane, W. V. "A Tale of Two Compartments: Interstitial Versus Blood Glucose Monitoring", Diabetes Technology \& Therapeutics, 11(S1), pp. S11-S16, 2009. https://doi.org/10.1089/dia.2009.0002

[24] The Diabetes Research in Children Network (DirecNet) Study Group "The Accuracy of the Guardian ${ }^{\circledR}$ RT Continuous Glucose Monitor in Children with Type 1 Diabetes", Diabetes Technology and Therapeutics, 10(4), pp. 266-272, 2008. https://doi.org/10.1089/dia.2007.0279

[25] Ricci, F., Moscone, D., Palleschi, G. "Ex Vivo Continuous Glucose Monitoring With Microdialysis Technique: The Example of GlucoDay", IEEE Sensors Journal, 8(1), pp. 63-70, 2008. https://doi.org/10.1109/JSEN.2007.912535

[26] Weinstein, R. L., Schwartz, S. L., Brazg, R. L., Bugler, J. R., Peyser, T. A., McGarraugh, G. V. "Accuracy of the 5-Day FreeStyle Navigator Continuous Glucose Monitoring System: Comparison with frequent laboratory reference measurements", Diabetes Care, 30(5), pp. 1125-1130, 2007. https://doi.org/10.2337/dc06-1602

[27] Kudo, H., Sawada, T., Kazawa, E., Yoshida, H., Iwasaki, Y., Mitsubayashi, K. "A flexible and wearable glucose sensor based on functional polymers with Soft-MEMS techniques", Biosensors and Bioelectronics, 22(4), pp. 558-562, 2006. https://doi.org/10.1016/j.bios.2006.05.006

[28] Lee, H., Song, C., Hong, Y. S., Kim, M. S., Cho, H. R., Kang, T., Shin, K., Choi, S. H., Hyeon, T., Kim, D. H. "Wearable/disposable sweat-based glucose monitoring device with multistage transdermal drug delivery module", 3(3), Science Advances, 3(3), Article Number: e1601314, 2017. https://doi.org/10.1126/sciadv.1601314

[29] Lansdorp, B., Ramsay, W., Hamid, R., Strenk, E. "Wearable Enzymatic Alcohol Biosensor", Sensors, 19(10), Article Number: 2380, 2019. https://doi.org/10.3390/s19102380

[30] Zheng, Z. Q., Yao, J. D., Wang, B., Yang, G. W. "Light-controlling, flexible and transparent ethanol gas sensor based on $\mathrm{ZnO}$ nanoparticles for wearable devices", Scientific Reports, 5(1), Article Number: 11070, 2015. https://doi.org/10.1038/srep11070

[31] Maity, D., Rajavel, K., Kumar, R. T. R. "Polyvinyl alcohol wrapped multiwall carbon nanotube (MWCNTs) network on fabrics for wearable room temperature ethanol sensor", Sensors and Actuators B: Chemical, 261, pp. 297-306, 2018. https://doi.org/10.1016/j.snb.2018.01.152

[32] Kim, J., Jeerapan, I., Imani, S., Cho, T. N., Bandodkar, A., Cinti, S., Mercier, P. P., Wang, J. "Noninvasive Alcohol Monitoring Using a Wearable Tattoo-Based Iontophoretic-Biosensing System", ACS Sensors, 1(8), pp. 1011-1019, 2016. https://doi.org/10.1021/acssensors.6b00356

[33] Sonner, Z., Wilder, E., Heikenfeld, J., Kasting, G., Beyette, F., Swaile, D., Sherman, F., Joyce, J., Hagen, J., Kelley-Loughnane, N., Naik, R. "The microfluidics of the eccrine sweat gland, including biomarker partitioning, transport, and biosensing implications", Biomicrofluidics, 9(3), Article Number: 031301, 2015.

https://oi.org/10.1063/1.4921039
[34] Schaefer, M., Schellenberg, M., Merle, U., Weiss, K. H., Stremmel, W. "Wilson protein expression, copper excretion and sweat production in sweat glands of Wilson disease patients and controls", BMC Gastroenterology, 8(1), Article Number: 29, 2008. https://oi.org/10.1186/1471-230X-8-29

[35] Mudgal, V., Madaan, N., Mudgal, A., Singh, R. B., Mishra, S. "Effect of Toxic Metals on Human Health", The Open Nutraceuticals Journal, 3, pp. 94-99, 2010. https://doi.org/10.2174/18763960010030100094

[36] Roohani, N., Hurrell, R., Kelishadi, R., Schulin, R. "Zinc and its importance for human health: An integrative review", Journal of Research in Medical Sciences, 18(2), pp. 144-157, 2013.

[37] Gao, W., Nyein, H. Y. Y., Shahpar, Z., Fahad, H. M., Chen, K., Emaminejad, S., Gao, Y., Tai, L. C., Ota, H., Wu, E., Bullock, J., Zeng, Y., Lien, D. H., Javey, A. "Wearable Microsensor Array for Multiplexed Heavy Metal Monitoring of Body Fluids", ACS Sensors, 1(7), pp. 866-874, 2016. https://doi.org/10.1021/acssensors.6b00287

[38] Nyein, H. Y. Y., Gao, W., Shahpar, Z., Emaminejad, S., Challa, S., Chen, K., Fahad, H. M., Tai, L. C., Ota, H., Davis, R. W., Javey, A. "A Wearable Electrochemical Platform for Noninvasive Simultaneous Monitoring of $\mathrm{Ca}^{2+}$ and $\mathrm{pH}^{\prime \prime}$, ACS Nano, 10(7), pp. 7216-7224, 2016. https://doi.org/10.1021/acsnano.6b04005

[39] Farahi, R. H., Ferrell, T. L., Guiseppi-Elie, A., Hansen, P. "Integrated electronics platforms for wireless implantable biosensors", In: 2007 IEEE/NIH Life Science Systems and Applications Workshop, Bethesda, MD, USA, 2007, pp. 27-30. https://doi.org/10.1109/LSSA.2007.4400876

[40] Zhou, W., Dai, X., Fu, T. M., Xie, C., Liu, J., Lieber, M. "Long Term Stability of Nanowire Nanoelectronics in Physiological Environments", Nano Letters, 14(3), pp. 1614-1619, 2014. https://doi.org/10.1021/n1500070h

[41] Sargolzaei, A., Yen, K. K., Abdelghani, M. N. "Control of Nonlinear Heartbeat Models under Time-Delay-Switched Feedback Using Emotional Learning Control", International Journal on Recent Trends in Engineering and Technology, 10(2), pp. 85-91, 2014.

[42] Lewitus, D. Y., Smith, K. L., Landers, J., Neimark, A. V., Kohn, J. "Bioactive agarose carbon-nanotube composites are capable of manipulating brain-implant interface", Journal of Applied Polymer Science, 131(14), Article Number: 40297, 2014. https://doi.org/10.1002/app.40297

[43] Basar, M. R., Malek, F., Juni, K. M., Idris, M. S., Saleh, M. I. M. "Ingestible Wireless Capsule Technology: A Review of Development and Future Indication", International Journal of Antennas and Propagation, 2012, Article ID: 807165, 2012. https://doi.org/10.1155/2012/807165

[44] Lewis, B. S. "The History of Enteroscopy", Gastrointestinal Endoscopy Clinics of North America, 9(1), pp. 1-11, 1999. https://doi.org/10.1016/S1052-5157(18)30216-2

[45] Iddan, G., Meron, G., Glukhovsky, A., Swain, P. "Wireless capsule endoscopy", Nature, 405(6785), pp. 417-418, 2000. https://doi.org/10.1038/35013140 
[46] Eliakim, R., Yassin, K., Shlomi, I., Suissa, A., Eisen, G. M. "A novel diagnostic tool for detecting oesophageal pathology: the PillCam oesophageal video capsule", Alimentary Pharmacology and Therapeutics, 20(10), pp. 1083-1089, 2004. https://doi.org/10.1111/j.1365-2036.2004.02206.x

[47] Li, C. Y., Zhang, B. L., Chen, C. X., Li, Y. M. "OMOM capsule endoscopy in diagnosis of small bowel disease", Journal of Zhejiang University: Science B, 9(11), pp. 857-862, 2008. https://doi.org/10.1631/jzus.B0820034
[48] Gheorghe, C., Iacob, R., Bancila, I. "Olympus capsule endoscopy for small bowel examination", Journal of Gastrointestinal and Liver Diseases, 16(3), pp. 309-313, 2007.

[49] Kim, H. M., Kim, Y. J., Kim, H. J., Park, S., Park, J. Y., Shin, S. K., Cheon, J. H., Lee, S. K., Lee, Y. C., Park, S. W., Bang, S., Song, S. Y. "A Pilot Study of Sequential Capsule Endoscopy Using MiroCam and PillCam SB Devices with Different Transmission Technologies", Gut and Liver, 4(2), pp. 192-200, 2010. https://doi.org/10.5009/gn1.2010.4.2.192 